\section{IN BRIEF}

\section{$\Rightarrow$ INCONTINENCE}

\section{RCT of group versus individual PFMT}

Individual pelvic floor muscle training (PFMT) is recommended for stress or mixed urinary incontinence in women, but its use is limited by cost and resources. The Group Rehabilitation or Individual Physiotherapy (GROUP) study included 362 women aged $\geq 60$ years with symptoms of stress or mixed urinary incontinence who, after an individual session in which they learnt how to contract the pelvic floor muscles, completed 12-week PFMT either as a group of 8 women $(n=178)$ or individually $(n=184)$. At 1 year, median percentage reduction in urinary incontinence episodes was $70 \%$ in women receiving individual PFMT versus $74 \%$ in those taking part in group-based PFMT. Use of group-based PFMT in clinical practice could help increase affordability and treatment availability for women with incontinence.

ORIGINAL ARTICLE Dumoulin, C. et al. Group-based vs individual pelvic floor muscle training to treat urinary incontinence in older women: a randomized clinical trial. JAMA Intern. Med. https://doi.org/10.1001/jamainternmed.2020.2993 (2020)

\section{$\Rightarrow$ BLADDER CANCER}

\section{ADXBLADDER test better than cytology for NMIBC follow-up monitoring}

ADXBLADDER, a urine test that detects MCM5 (presence of which in urine sediment can indicate the presence of a bladder tumour), is superior to urine cytology for detecting recurrence of non-muscle-invasive bladder cancer (NMIBC). A multicentre prospective, blinded study collected urine from patients before cystoscopy and compared cytology and ADXBLADDER results with cystoscopy diagnosis. ADXBLADDER had a sensitivity of $51.9 \%$, a specificity of $66.4 \%$ and a NPV of $92 \%$, with a sensitivity of $44.1 \%$ and $58.8 \%$ for low-grade and high-grade recurrences, respectively. Cytology sensitivity was $16.7 \%$, specificity was $98 \%$ and NPV was $90.7 \%$, with a sensitivity of $17.6 \%$ for both low-grade and high-grade recurrences. Thus, detection of both low-grade and high-grade recurrences by ADXBLADDER is superior to that of urine cytology, and the test could provide a reliable alternative to urine cytology in NMIBC follow-up monitoring.

ORIGINAL ARTICLE Gontero, P. et al. Comparison of the performance of ADXBLADDER test and urinary cytology in the follow up of non-muscle invasive bladder cancer: a blinded prospective multicentric study. BJU Int. https://doi.org/10.1111/bju.15194 (2020)

\section{$\Rightarrow$ INFECTION}

\section{3-monthly HIV screening is best for young MSM}

Twenty per cent of new HIV infections in the USA arise in young men (aged 13-24 years) who have sex with men (YMSM); however, $>50 \%$ of YMSM with HIV do not know their HIV status. Mathematical simulation was used to assess clinical benefit and cost-effectiveness of yearly, 6-monthly and 3-monthly screening strategies, in addition to the status quo (SQ), in high-risk YMSM aged from 15 years. Published data (YMSM-specific when available) were used for the model and projected outcomes included CD4 count at diagnosis, primary HIV transmissions from age 15-30 years, quality-adjusted life expectancy, costs and incremental cost-effectiveness ratios. All strategies increased projected CD4 at diagnosis and quality-adjusted life expectancy from age 15 compared with SQ, and also increased discounted lifetime cost for the entire population. Screening at 3-monthly intervals was cost-effective compared with SQ and reduced primary transmissions by $40 \%$ in YMSM aged up to 30 years.

ORIGINAL ARTICLE Neilan, A. M. et al. Cost-effectiveness of frequent HIV screening among high-risk young men who have sex with men in the United States. Clin. Infect. Dis. https://doi.org/10.1093/cid/ciaa1061 (2020)

\title{
NRG1 can promote antiandrogen resistance
}

NRG1 secreted by cancer-associated fibroblasts (CAFs) can promote antiandrogen resistance in prostate cancer, according to a new study.

Second-generation antiandrogens have improved survival of patients with prostate cancer, but acquired resistance to hormone therapy is a major problem. Researchers have shown that the tumour microenvironment - stroma and inflammatory cells - promotes drug resistance in some cancers.

A reactive stroma tumour microenvironment has been demonstrated in several cancers and is likely to promote tumorigenesis. In their study, Zhang et al. showed that reactive stroma score was higher in prostate tumour samples than in normal prostate gland. Higher-grade tumour samples (Gleason score $\geq 7$ ) had higher reactive stroma scores than lower-grade tumour samples (Gleason score $<7$ ).

The researchers modelled the tumour-stroma interaction using a patient-derived xenograft model and found that a cell line derived from this model had a population of murine CAFs. They purified the human cancer (epithelial) cells from the murine CAFs. Isolation of the human cancer epithelial cells from the murine CAFs led to delay of bicalutamide resistance (152 days versus 48 days in the mixed cell population). The authors hypothesized that CAFs can promote antiandrogen resistance.

The researchers demonstrated that the growth-promoting effects of CAFs on cancer cell growth during antiandrogen treatment occur through secretion of CAF-derived soluble factors. They used biochemical fractionation to search for key proteins responsible for antiandrogen resistance and found that neuregulin 1 (NRG1) protein expressed by tumour-infiltrating CAFs promotes resistance in cancer cells through activation of HER3.

In vivo mouse xenograft models were used to explore the physiological relevance of the finding that paracrine NRG1-HER3 signalling drives resistance. Zhang et al. found higher levels of NRG1 (in stroma) and phospho-HER3 (in tumour cells) in lysates from prostate cancer xenografts grown in castrated mice than in intact mice.

Treatment of castration-resistant prostate cancer xenografts with a blocking antibody to HER3 or a HER2 kinase inhibitor (neratinib) resulted in tumour growth inhibition. NRG1-neutralizing antibodies also had antitumour activity, given alone or in combination with neratinib. In a castration-sensitive model of prostate cancer, NRG1 blockade enhanced the antitumour effect of castration, either alone or in combination with neratinib.

The researchers used immunohistochemical assays to examine NRG1 expression in 43 patients with localized prostate cancer who underwent radical prostatectomy, 23 of whom were on neoadjuvant androgen deprivation therapy (ADT). NRG1 staining was present in 0 of 20 patients not receiving ADT at prostatectomy and 5 of 23 (22\%) patients who received ADT before prostatectomy. Zhang et al. report that ADT induced NRG1 expression in tumour-associated stromal cells of primary prostate cancers and that an increased NRG1 level promotes resistance to ADT.

Further work in patients with castration-resistant prostate cancer showed that patients with tumour NRG1 activity levels in the top $50 \%$ showed a poorer response to second-generation antiandrogen therapy, with a significantly shorter time to progression on enzalutamide or abiraterone, than patients with NRG1 activity in the bottom $50 \%$.

Rebecca Kelsey

ORIGINAL ARTICLE Zhang, Z. et al. Tumor microenvironment-derived NRG1 promotes antiandrogen resistance in prostate cancer. Cancer Cell https://doi.org/10.1016/j.ccell.2020.06.005 (2020) 